\title{
A New Approach to Volunteer Cloud Computing
}

\author{
Ruchika Saini ${ }^{1}$, Pawan Prakash ${ }^{1}$ \\ ${ }^{1}$ (Dept. Of Computer Science and Engineering, Gyan Vihar University, Jaipur, Rajasthan, India)
}

\begin{abstract}
Volunteer Cloud Computing is based on the concept where highly distributed non-dedicated resources are harnessed to build a cloud so as to offer cloud services. As volunteer clouds are allowed to communicate with each other and with other commercial clouds also, it's necessary to implement an enhanced interoperable environment. In this paper we propose an XMPP based messaging middleware architecture that can help in implementing such an environment.
\end{abstract}

Keywords: cloud computing, commercial cloud, interoperability, middleware architecture, volunteer cloud.

\section{Introduction}

The concepts of cloud computing and volunteer computing are combined to form volunteer cloud computing. In volunteer cloud computing idle or non-dedicated resources [1] that are not designed to be cloud infrastructure are harnessed so as to provide cloud capabilities or services. By volunteer cloud computing [2] [3] [4] users and contributors can experience the impact of cloud computing by allowing users to share any type of service be it physical resources, software resources, applications.

It offers some advantages [3] [5]:

- harnessing idle resources

- reduce the cost

- reduce overall power consumption

Non dedicated resources are used to constitute volunteer contributors clouds that can communicate with each other and also with other commercial clouds. So in order to achieve interoperability between volunteer contributor cloud and commercial cloud, and to deal with moving and reallocating data, we propose an XMPP based messaging middleware architecture.

This paper is structured as follows: the next section presents what is the need to establish a communication between volunteer clouds and commercial clouds and challenges for achieving the same, than we discuss literature review. Thirdly we present our proposed work and some possible solutions to these challenges. Finally we end up with a conclusion and future work.

Scientific or research projects that cannot be able to have commercial cloud services can use volunteer clouds where contributors and users voluntarily share their resources. For short and long term projects, our traditional IT companies have to spend a lot of time in deploying new hardware and software resources, by procuring and purchasing these new resources into their infrastructure.

Volunteer cloud computing offers cloud services that are based on non dedicated resources without charging and a way to cut down IT cost so that companies can benefit from the well planned budget and can make the best use of it. We can make use of volunteer clouds, if a company wants to endeavor a project for a short duration of time. So it's time to move complex solutions to a volunteer cloud that can offer comparatively much faster response to the needs and requirements of business and can help companies lower overhead.

\section{Challenges}

Important challenges [6] that should be taken into consideration in order to achieve better communication between volunteer clouds and commercial clouds are as follows:

One directional communication: it's one of the biggest challenge. So our middleware should be such that it can help to establish a proper communication.

Latency: interaction between nodes is somewhat slower than interaction between clouds, so a middleware is required that can perfectly handle such delays.

Availability: resources should be made available easily as per the needs of the users.

Security: in order to secure the communication, we need some effective mechanism to deal with authentication, data protection and integrity.

Compatibility problem among different cloud infrastructure.

\section{Literature Review}

This section presents the following related work: volunteer cloud computing architecture and cloud@home architecture. Volunteer cloud computing architecture [3] contains three layers: a service layer, virtual layer and 
physical layer. Service layer, in this layer services are provided to customers via an interface which is based on SOA approach. Next layer is the virtual layer it provides multiple functionalities like task management and QoS management. Last layer is the physical layer that deals with resource aggregation, allocation and monitoring.

Cloud@home [7] [8] deals with the reuse of domestic computing resources in order to deploy voluntary clouds. By cloud@ home users and contributors can share their resources and services of any kind. This logical abstract model defines some layers: software environment, software infrastructure, software kernel and firmware/hardware. Software environment layer deals with interoperability among clouds and also responsible for checking services like availability and reliability. Next layer, software infrastructure, it provides two basic services to end users: execution and storage services. Third layer software kernel, in order to manage execution and storage services at the above layer software kernel provides some mechanism and tools. Final layer firmware/hardware, for the implementation of execution and software services this layer provides the physical hardware resources to the upper layer.

Even after these approaches, some challenges are still left that should be taken into account in order to achieve interoperability.

\section{Proposed Work}

We now implement an XMPP protocol over middleware layer so as to form XMPP based messaging middleware layer that can provide enhanced interoperability and can help towards a solution for the challenges mentioned above.

\subsection{Xmpp}

Extensible messaging and presence protocol (XMPP) is an open protocol for real time communications based on XML (extensible markup language) that works as a communication protocol over message oriented middleware. XMPP based messaging middleware architecture provides a wide range of services that deals with the issues that we have discussed earlier are interoperability, availability, faster two way communication, flexibility, security.

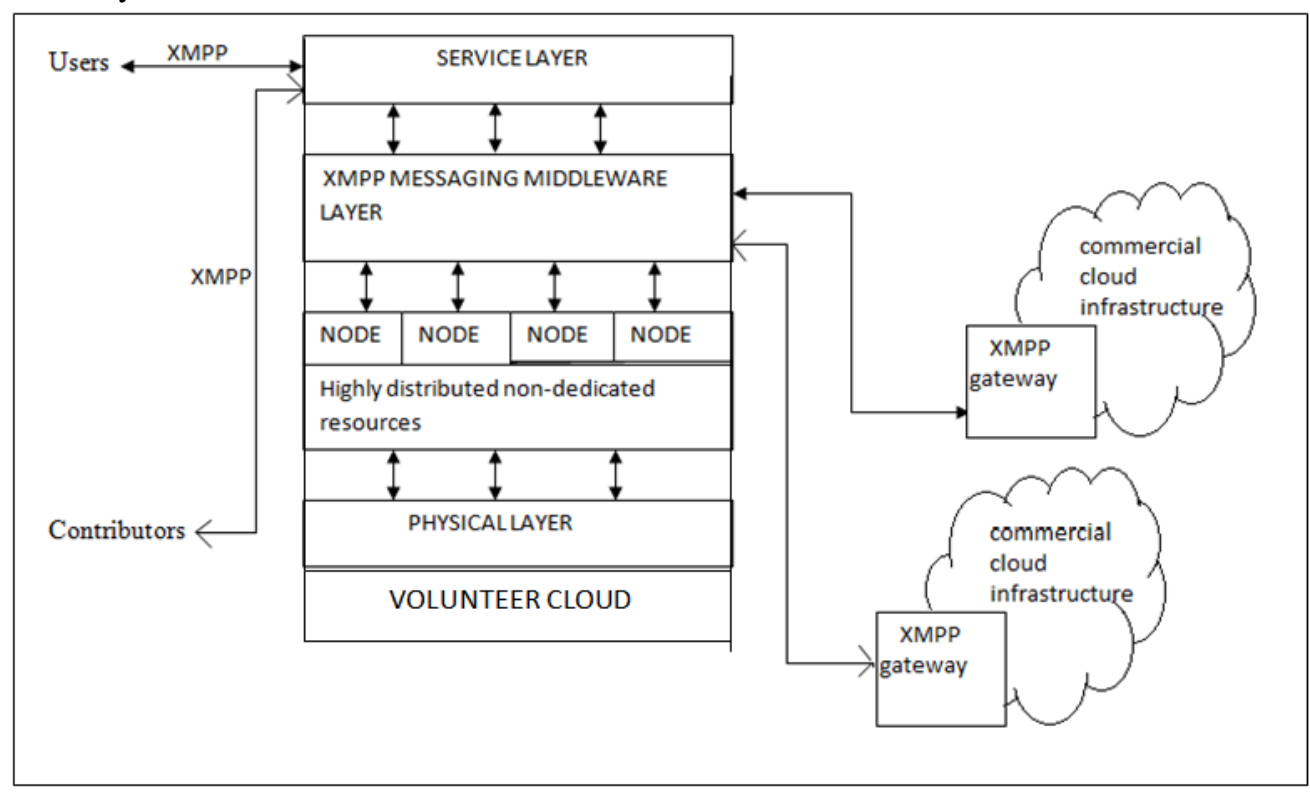

Fig.1 XMPP based messaging middleware architecture

For clouds to interact with each other, a common format for messaging is used to allow the resources to interoperate with each other and manifest how their services can be utilized. On different cloud network, if resources are not implemented by XMPP and if the communication is to be established, then there is XMPP gateway that can convert XMPP to a foreign protocol. Any user who wants to communicate with the user on the other network, that user will first have to register with one of the gateways for the authentication purpose and then only they can start the communication.

XMPP presence server receives presence information from XMPP clients. Presence state, addressing information and protocols are the present data distributed in XML streams. So to manage the availability of volunteer cloud entities, presence information is used and incoming and outgoing requests are handled by XMPP servers. Further load balancer is used to prioritize the incoming requests. So this XMPP based messaging middleware architecture is used for resource monitoring, and to improve load balancing so as to increase availability. 
With emerging cloud computing technologies, XMPP is an ideal middleware protocol. Cloud services often use SOAP and some other HTTP-based protocols, but the problem with these protocols, is that they all offer one way communication that makes the services non real time, won't extend. There is another problem of long polling with HTTP based protocols, it means that server has to wait until it receives an update and as soon as it receives an update, the server sends the response and then only client can send further request. In this regard, we make use of XMPP as it offers faster and easy two way communication and also eliminates long polling. XMPP is also designed to be scaled and it provides SASL and TLS mechanism in order to provide robust security.

\section{Conclusion}

In conclusion, XMPP based messaging middleware architecture overcomes the major challenges that we have discussed so as to achieve enhanced communication between volunteer contributors clouds and other commercial clouds infrastructure. XMPP offers a wide range of services like two way communication, eliminates long polling, enhanced interoperability, availability and many more that can help towards a solution for these challenges and this is the reason that makes XMPP the most suitable communication protocol. Our future work involves implementing mechanisms for security in order to make our communication more secure.

\section{ACKNOWLEDGMENTS}

We would like to take this opportunity to thank \& express our special gratitude to Mr. Polash Bora for his support and help. Last but not the least we wish to express our gratitude to God Almighty for His abundant blessings without which this work would not have been successful.

\section{References}

[1] A. Andrzejak, D. Kondo, and D. P. Anderson, "Exploiting non dedicated resources for cloud computing," 2010 IEEE Network Operations and Management Symposium - NOMS 2010, pp. 341-348, 2010.

[2] A. Marosi, J. Kovács, and P. Kacsuk, "Towards a volunteer cloud system," Future Generation Computer Systems, Mar. 2012

[3] Abdulelah Alwabel, Robert Walters and Gary Wills "towards an architecture for IaaS volunteer cloud" digital research 2012.

[4] Fernando Costa, Luis Silva, Michael Dahlin, " volunteer cloud computing: mapreduce over the internet", Parallel and Distributed Processing Workshops and Phd Forum (IPDPSW), 2011 IEEE International Symposium on.

[5] D. Kondo, B. Javadi, P. Malecot, F. Cappello, and D. Anderson, "Costbenefit analysis of Cloud Computing versus desktop grids", in Proceedings of the 2009 IEEE international Symposium on Parallel \& Distributed Processing, pp. 1-12, May 2009

[6] T. Dillon, C. Wu, and E. Chang, "Cloud computing: Issues and challenges," in 201024 th IEEE International Conference on Advanced Information Networking and Applications, 2010, pp. 27-33.

[7] Vincenzo D. Cunsolo, Salvatore Distefano, Antonio Puliafito and Marco Scarpa, "Cloud@Home: Bridging the Gap between Volunteer and Cloud Computing", in ICIC'09 Proceedings of the 5th international conference on Emerging intelligent computing technology and applications, 2009.

[8] V. D. Cunsolo, S. Distefano, A. Puliafito, and M. Scarpa, "Applying Software Engineering Principles for Designing Cloud@Home," 2010 10th IEEE/ACM International Conference on Cluster, Cloud and Grid Computing, pp. 618-624, 2010. 